Service social

\title{
La réforme de l'aide sociale. Enjeux et propositions concernant la lutte à la pauvreté
}

\section{Camil Bouchard}

Volume 44, numéro 3, 1995

Pauvreté

URI : https://id.erudit.org/iderudit/706709ar

DOI : https://doi.org/10.7202/706709ar

Aller au sommaire du numéro

\section{Éditeur(s)}

École de service social de l'Université Laval

ISSN

1708-1734 (numérique)

Découvrir la revue

Citer cet article

Bouchard, C. (1995). La réforme de l'aide sociale. Enjeux et propositions concernant la lutte à la pauvreté. Service social, 44(3), 115-144.

https://doi.org/10.7202/706709ar

\section{Résumé de l'article}

Le débat autour de l'aide sociale, s'il prend parfois l'allure d'un débat sur les finances publiques, est d'abord un "' débat autour d'une lente construction de la démocratie et de la citoyenneté "' (Noël, 1996). Certes, la prévention et la diminution de la pauvreté passent par une meilleure redistribution des revenus et par la création d'emplois, notamment pour les jeunes et au niveau local. Mais elles passent aussi par un enrichissement du rôle de citoyen et par une participation plus grande à l'exercice individuel et collectif de la démocratie. Les possibilités de se développer dépendent non pas exclusivement de la motivation des individus mais aussi, et surtout, du fait d'une reconnaissance collective et sociale du problème de la pauvreté et de ses effets. Ces principes représentent globalement les fondements sur lesquels s'appuie le rapport Chacun sa part soumis à l'attention du gouvernement du Québec (en mars 1996) par un groupe majoritaire de membres du Comité externe de la réforme de la sécurité du revenu. Pour les signataires du rapport majoritaire (Chacun sa part), on ne peut demander aux personnes les plus pauvres de faire seules les frais d'une réforme et encore moins d'un redressement des finances publiques. Il y a là des enjeux de solidarité, d'équité, et ultimement de démocratie.
Ce document est protégé par la loi sur le droit d'auteur. L'utilisation des services d'Érudit (y compris la reproduction) est assujettie à sa politique d'utilisation que vous pouvez consulter en ligne.

https://apropos.erudit.org/fr/usagers/politique-dutilisation/ 


\section{La réforme de l'aide sociale. Enjeux et propositions concernant la lutte à la pauvreté}

Camil BOUCHARD

Professeur et chercheur à I'Université du Québec à Montréal (Département de psychologie, secteur de la psychologie communautaire) Membre fondateur du Laboratoire de recherche en écologie humaine et sociale Université du Québec à Montréal'

La pauvreté engendre des risques d'exclusion à trois niveaux aussi bien pour les individus que pour les communautés: exclusion économique, exclusion sociale et exclusion politique. Parce que la pauvreté et les effets qu'elle engendre menacent les valeurs fondamentales de solidarité, d'équité et de démocratie, on ne peut la contrer que sur le seul terrain économique. L'accès à un meilleur revenu certes, mais aussi une probabilité plus grande de participation à leur propre cheminement $d^{\prime}$ insertion et aux décisions institutionnelles qui les touchent personnellement, fait partie des stratégies qui concernent le bien-être, le développement et la citoyenneté des personnes démunies. Ces stratégies ont été largement approfondies dans Chacun sa part. Le présent article en fait brièvement état. II s'articule autour de 
six objectifs implicitement poursuivis par le rapport: 1) prévenir le surplace et la dépréciation de soi ; 2) éviter que le fatalisme et la résignation $\mathrm{n}^{\prime}$ accablent les communautés; 3 ) éviter que des vies entières ne s'installent dans la pauvreté ; 4) prévenir l'enlisement dans la pauvreté ; 5) prévenir la mise à l'écart des groupes particuliers; 6) prévenir l'érosion de la solidarité.

\section{PRÉVENIR LE SURPLACE ET LA DÉPRÉCIATION DE SOI}

Le régime actuel d'insertion professionnelle fondé sur une approche d'employabilité (loi 37) présente plusieurs lacunes. Géré principalement en fonction de la réduction des effectifs et par là des barèmes, le système: 1 ) ne répond pas à la demande ;2) n' offre à peu près rien à ceux qui ne participent pas; 3) conduit peu de gens vers l'emploi ; 4) décourage la majorité de personnes qui participent de bonne foi et répondent aux attentes sans pour autant obtenir un emploi ; 5) met I'accent sur le volume d'activités et la réduction du nombre de personnes recevant le barème des disponibles;6) manque de transparence et ne permet pas aux prestataires de prendre en main leur situation et de définir leur parcours en fonction de leurs champs $d^{\prime}$ intérêt, de leurs aspirations et de leurs projets ; 7) dirige les personnes vers la formation en institution sans tenir compte du fait qu'elles ont souvent eu des difficultés dans le système scolaire; 8 ) crée des passerelles vers l'emploi sans objectifs précis, sans formation véritable et sans débouchés clairs; 9) crée des emplois sans accorder aux personnes un salaire véritable, le statut de salarié et la protection de la Loi sur les normes de travail; 10) a des effets de substitution en déplaçant des emplois qui auraient été créés de toute façon; 11) tient peu compte de l'utilité collective et sociale des mesures et des emplois créés ; 12) impose peu de contraintes aux différents partenaires qui ne considèrent souvent les mesures que sous l'angle de financement de leurs activités; 13) met l'accent sur les incitations financières et les contrôles, alors que la formation et la recherche d'emploi reposent aussi sur les motivations intrinsèques des individus; 14) pose le problème en termes d'employabilité individuelle, alors qu'il s'agit avant tout d'un problème de manque global d'emplois et de barrières à l'emploi dans l'ensemble de l'économie.

Ce diagnostic ne devrait pas nous amener à conclure qu'il faut abandonner toute démarche de formation personnelle et d'intégration au marché du travail. II faut plutôt repenser le système sur la base de principes plus justes et plus adéquats. II n'y a pas de solution administrative simple à la pénurie d'emplois, et l'approche centrée sur 
l'employabilité est une réponse simple et inadéquate à un problème complexe. Les meilleures mesures d'orientation, de formation, $d^{\prime}$ insertion et d'emploi laisseront toujours des gens en dehors du marché du travail régulier. II faut donc replacer les choses dans une perspective plus large, et faire du parcours vers l'emploi une dimension nouvelle de notre système de protection sociale. La notion de parcours vers l'emploi implique en effet beaucoup plus qu'une procédure ponctuelle de rehaussement de l'employabilité. II s'agit plutôt d'inscrire un nouveau droit à l'intérieur de la sécurité du revenu, le droit à l'intégration sociale et économique. En reconnaissant ce droit, le Québec s'engagerait à ouvrir des avenues aux personnes les plus démunies, avenues débouchant autant que possible, mais pas toujours, sur l'intégration au marché du travail. On peut concevoir, par exemple, que certaines personnes voudront se prévaloir des programmes d'alphabétisation, même si elles n'ont que peu de chances d'accéder à un emploi (à cause de contraintes sévères ou de leur âge). Pour ces personnes, l'accès à de telles mesures, et plus fondamentalement la possibilité de bâtir un projet personnel et d'en parler, serait une voie d'intégration sociale légitime et valorisante ${ }^{2}$. II faut toujours garder à l'esprit que les mesures en question concernent très directement la vie des gens les plus pauvres, les moins puissants et, souvent, les plus fragiles d'entre nous. II importe donc de faire preuve d'une grande prudence et de beaucoup d'humilité dans nos propositions et dans nos tentatives. À partir de ces principes, les signataires de Chacun sa part considèrent qu'il faut:

- abandonner la notion d'employabilité et parler plutôt de parcours vers l'emploi ;

- mettre la personne au centre du processus et construire des parcours cohérents et continus et définis sur une base volontaire;

- faire en sorte que toute personne admise à la sécurité du revenu, y compris les personnes ayant des contraintes sévères à l'emploi, ait accès à un minimum de services d'accompagnement et d'encadrement, et que ces mêmes services soient également accessibles, sur demande, à celles qui ne sont pas à la sécurité du revenu;

- intégrer les mesures actuelles à la formation, aux politiques actives et aux emplois standards, qui s'adressent à l'ensemble des citoyens, et réaliser cette intégration sur le plan local et régional;

- simplifier le système, harmoniser les normes et les attentes bureaucratiques aux objectifs énoncés, et garantir une information claire et un traitement équitable aux personnes; 
- travailler en partenariat avec le secteur public, le secteur privé et le secteur communautaire, et le faire à partir de règles exigeantes mais claires, qui soient justes envers les personnes et les organisations et qui cherchent à promouvoir l'utilité collective ;

- associer les mesures au marché du travail, et reconnaître que tout travail mérite une reconnaissance, un salaire et une protection légale normale.

Les auteurs recommandent aussi :

- de reconnaître la dimension éminemment collective et sociale de l'insertion sociale et professionnelle, d'offrir des options réalistes et d'appuyer les démarches des personnes dans leur parcours vers l'emploi;

- de développer des mesures d'insertion sociale et professionnelle cohérentes, qui permettent à chaque personne de définir un parcours personnel vers l'emploi qui soit continu et progressif, et qui tienne compte des expériences de la personne, de ses centres d'intérêt et de ses projets ;

- d'inscrire le parcours de chaque personne à l'intérieur d'un plan $d^{\prime}$ insertion sociale et professionnelle, plan pouvant inclure des démarches d'insertion sociale, de recherche d'emploi, de formation, d'insertion professionnelle, et d'emploi. Ce plan devrait être défini conjointement par la personne et son conseiller et il devrait donner lieu à une entente signée engageant les deux parties ;

- de poursuivre l'accompagnement et l'encadrement des personnes tout au long de leur démarche;

- de simplifier le système actuel dans un programme en quatre volets: Orientation, Formation, Insertion et Emploi ${ }^{3}$;

- d'éliminer les différents barèmes de statut actuellement offerts à la Sécurité du revenu pour convertir les sommes épargnées en mesures actives;

- de rapatrier au Québec la gestion complète de I'assuranceemploi afin d'intégrer le versement des prestations, le placement et les politiques actives et d'élaborer une politique active et intégrée du marché du travail. Cela constitue une pièce maîtresse dans la recherche $d^{\prime}$ une solution à la pauvreté. De fait, cela permettrait notamment d'utiliser une partie des surplus enregistrés au régime d'assurance-chômage dans un Fonds de régulation des dépassements conjoncturels qui mettrait le budget de 
la Sécurité du revenu à l'abri des coupures dans les moments où il faut au contraire offrir davantage aux citoyens.

\section{ÉVITER QUE LE FATALISME ET LA RÉSIGNATION N'ACCABLENT LES COMMUNAUTÉS}

\section{Dessiner un système dynamique et démocratique}

La capacité d'une organisation à multiplier les occasions où la contribution de ses membres est attendue et indispensable augmente la probabilité que les membres s'y identifient, y contribuent et elle évite qu'ils ne tombent dans l'indifférence, le fatalisme ou la résignation. Cela est d'autant plus vrai lorsqu'il s'agit de groupes menacés $d^{\prime}$ exclusion. Une telle capacité est plus facile à réaliser dans un petit territoire; la proximité des instances responsables et imputables permet davantage d'accès aux pouvoirs, facilite le décodage et la compréhension des règles et des modes de fonctionnement et diminue le sentiment d'impuissance ressenti devant la bureaucratie. Enfin, une gestion plus locale permet de mettre à profit la connaissance que les intervenants ont des ressources, des possibilités, des réseaux d'information dans leur environnement. On peut alors compter sur une valeur ajoutée émanant de l'interaction des acteurs locaux du monde des affaires, de l'éducation, de la santé et des services sociaux, de la sécurité du revenu, de l'emploi, du loisir et des groupes communautaires.

Suivant ces affirmations, les signataires de Chacun sa part recommandent très fortement que :

- des Centres locaux d'emploi (CLE) soient formés à partir de la fusion des centres Travail-Québec, des Centres d'emploi du Canada et des points de services de la Société québécoise de développement de la main-d'œuvre ${ }^{4}$;

- chaque centre local d'emploi se dote d'un Conseil des partenaires locaux pour l'emploi qui veillera à la bonne administration du Centre et aura pour principale mission la définition et la mise en œuvre d'un Plan local d'action pour l'emploi (PLACE) révisé annuellement ;

- soient mis en place des comités paritaires de fonctionnaires, $d^{\prime}$ 'usagers du système et d'experts en alphabétisation et en éducation populaire pour valider les parcours bureaucratiques et pour améliorer la gestion à mesure que les problèmes se présenteront. 


\section{Revoir les mécanismes d'arbitrage et de participation}

Même avec un bon système et des intervenants bien intentionnés, des différences d'intérêt ou d'interprétation se manifesteront inévitablement entre groupes d'intérêts divergents et entre personnes dont l'accès au pouvoir est forcément inégal. Les signataires de Chacun sa part pensent que:

- il faut appuyer la mise sur pied de Comités d'usagers ${ }^{5}$ au sein même du réseau des CLE. Ces comités d'usagers devraient avoir une présence dans le réseau tout comme les syndicats ont une présence dans l'entreprise ou que les associations étudiantes ont une présence dans les établissements d'enseignement. Les comités d'usagers proposés offriraient des services de conseil et $d^{\prime}$ appui et de représentation, participeraient aux exercices d'évaluation du système local et pourraient aussi servir de base pour différentes activités sociales et communautaires. Ils siégeraient aux Conseils des partenaires locaux pour l'emploi et, sur une base fédérative, à la Société québécoise de développement de la main-d'œuvre. Ils pourraient être en partie autofinancés par une déduction à la source. II s'agit ici de donner une voix aux usagers, de protéger leurs droits et d'assurer l'équité du processus d'aide.

\section{Développer l'emploi localement}

Si des mesures macro-économiques et législatives peuvent créer les conditions favorables au développement de l'emploi (Chacun sa part en fait largement état), il n'en reste pas moins que $c^{\prime}$ est au niveau local que la débrouillardise et la créativité sont requises pour explorer les avenues qui s'imposent. De fait, on a assisté depuis le début des années 80 à un extraordinaire effort de développement local. En milieu rural, cet effort s'est organisé autour des Services d'aide au développement des collectivités et des Services d'aide aux entreprises. En milieu urbain, il s'est beaucoup développé autour des Corporations de développement économique et des Corporations de développement économique communautaire (CDEC). Il faut appuyer cette énergie déployée à résoudre ensemble et localement des problèmes vécus localement; plusieurs des recommandations de Chacun sa part vont dans ce sens. Les signataires du rapport font aussi une large place à la création locale d'emplois d'utilité collective. 


\section{Développer les emplois d'utilité collective}

On pourrait craindre que les emplois d'utilité collective condamnent les travailleuses et travailleurs à des emplois bas de gamme et mal payés; les emplois d'utilité collective ont trop souvent été associés au Québec aux programmes d'employabilité. Cependant, des emplois de qualité ont souvent été développés sur une base d'utilité collective, par exemple dans les garderies sans but lucratif ${ }^{6}$. Pour que le statut de travailleur prenne tout son sens, ces nouveaux emplois doivent être plus que des emplois d'insertion et être le plus possible accessibles sans restrictions liées au statut ${ }^{7}$. Les zones de développement des emplois d'utilité collective $n^{\prime}$ ont pas encore été toutes recensées, mais une synthèse des énoncés sur le sujet donnerait pour l'heure la liste suivante: des réponses préventives pour améliorer la santé, la sécurité, l'environnement, la cohésion sociale, pour lutter contre la pauvreté, contre le décrochage, contre l'exclusion; des services domestiques liés à un taux moindre d'institutionnalisation; des services d'accompagnement et d'aide aux transitions de la vie; des services palliant les besoins et le manque de temps et de disponibilité des travailleuses et des travailleurs, ce qui intègre dans l'économie formelle des tâches traditionnellement assumées de façon informelle (garderie, cuisine collective, entretien, réparations, couture); des travaux d'aménagement et d'infrastructures améliorant la qualité de vie ou les attraits d'une communauté, d'une localité, d'une région; des services de soutien à la défense des droits, à l'éducation civique, économique, démocratique, à la construction de la solidarité; des contributions artistiques pour donner accès au beau; toute autre initiative estimée utile par la communauté.

L'ensemble des propositions avancées dans Chacun sa part ouvre de nombreuses possibilités de création d'emplois dans le champ de l'économie sociale: 1 ) un meilleur accès aux garderies pourrait conduire à la création de 2400 emplois d'aides-éducatrices; 2) le soutien nécessaire aux écoles primaires et secondaires en milieu appauvri pourrait aboutir à un programme de création d'emplois de soutien en appui aux enseignants. Il suffirait qu'on considère un ratio $d^{\prime}$ 'une personne pour 200 enfants et jeunes en milieu appauvri pour créer 1100 nouveaux emplois à l'échelle du Québec;3) de même, la réorientation des CTQ en Centres locaux d'emploi ne sera viable que si le milieu peut appuyer de façon considérable les efforts des conseillers des centres locaux. Les fonctions d'orientation, de formation, d'insertion et d'accès à l'emploi pourraient ouvrir la porte à 2250 emplois dans les organismes communautaires associés à cette mission; 4) une intensification des efforts d'éducation économique 
s'avérera également nécessaire pour aider les personnes à préserver à leur avantage la mince marge de manœuvre financière que leur fournit la sécurité du revenu. Plusieurs centaines de postes pourraient ainsi être créés (par exemple un emploi pour chaque millier d'adultes à la sécurité du revenu équivaudrait à 550 emplois) au niveau du suivi budgétaire, de la promotion de formules de crédit solidaire pour faire échec au crédit facile à haut taux d'intérêt, de l'éducation à la consommation.

Dans la mesure où les emplois créés dans le cadre du développement d'une économie sociale répondent à des besoins sociaux locaux et qu'ils sont de véritables emplois ${ }^{8}$ (durables, accessibles, de qualité, non substitutifs, soumis aux normes du travail), les signataires de Chacun sa part pensent que:

- le ministère de l'Emploi et de la Solidarité devrait assumer le leadership du développement des emplois d'utilité collective et 's'assurer, dans cette démarche, de la collaboration des nombreux ministères dont les missions les désignent comme partenaires privilégiés de ce chantier;

- le ministère de l'Emploi et de la Solidarité devrait prévoir la transformation graduelle d'une partie de ses budgets de transfert aux personnes en budgets de programmes d'emplois d'utilité collective. Même si ces emplois doivent être ouverts à tout le monde, il serait logique de s'assurer qu'ils intègrent un bon nombre de personnes en situation de chômage de courte ou de longue durée. Ces emplois ne devront toutefois pas servir à occuper inutilement des personnes, à les forcer à un travail de seconde zone ou à les tenir loin du marché de l'emploi.

\section{ÉVITER QUE DES VIES ENTIÈRES NE S'INSTALLENT DANS LA PAUVRETÉ}

\section{Offrir des services de garde facilement accessibles}

La fréquentation d'une garderie de qualité devrait représenter une priorité pour tous les enfants pauvres ou très pauvres dont les parents occupent ou non un emploi. La recherche démontre clairement les avantages que tirent les enfants de milieux défavorisés de la fréquentation assidue et intensive de programmes de stimulation offerts en bas âge ${ }^{9}$. Les efforts d'accompagnement de l'enfant jusqu'au seuil de l'école en l'insérant dans un réseau social d'enfants et d'adultes, et en assurant la participation active des ses parents dans ce réseau, 
représentent sans doute une des meilleures assurances contre le décrochage scolaire et contre la reproduction du scénario de la pauvreté. La recherche montre aussi qu'un des principaux motifs $d^{\prime}$ abandon, par les mères, des programmes d'insertion économique offerts dans le cadre de l'aide de dernier recours serait précisément la non-disponibilité des services de garde ${ }^{10}$. L'ensemble de ces considérations, notamment celles concernant le soutien au développement cognitif, social et affectif de l'enfant, nous ont amenés à proposer que :

- l'exonération totale (gratuité) devrait être accordée pour le temps complet à tout enfant entre 6 mois et 5 ans dont le ou les parents (cohabitant) répondent au critère de revenus insuffisants et sont inscrits sur le marché du travail ou dans une démarche d'insertion économique ou sociale. L'exonération totale devrait être accordée pour le demi-temps à tout enfant entre 6 mois et 5 ans (exclus) dont le ou les parents répondent au critère de revenu, mais ne sont pas sur le marché du travail et ne participent pas à une démarche d'insertion sociale ou économique et inscrivent leur enfant à une garderie régie au moins cinq demijournées par semaine. Ces garderies offrent les services d'une aide-éducatrice chargée de rendre compte régulièrement du développement de l'enfant aux parents.

Certaines considérations devraient être prises en considération dans I'application de cette recommandation. Nous en mentionnons ici quelques-unes: 1) une structure de taux de réduction permettant d'exonérer les parents déclarant un revenu supérieur aux seuils de revenus insuffisants devrait être mise en place afin d'éviter le problème d'un écart trop brutal entre l'aide accordée aux familles sous les seuils et celles immédiatement au-dessus de ces seuils ${ }^{11}$; 2) les services de garde en halte-garderie devraient être reconnus et régis. Ils devraient être offerts gratuitement à tous les bébés dont les parents répondent aux critères de revenus à condition d'une utilisation minimale d'une demi-journée par semaine ; 3) par souci de continuité dans les efforts, les commissions scolaires devraient réserver une petite partie de leurs taxes pour assurer la gratuité des services de garde aux élèves du primaire ; 4) on ne devrait ménager aucun effort pour tenter de convaincre les parents de donner à leur enfant l'occasion de fréquenter une garderie sur une base régulière; 5) la constitution d'un Fonds de création d'emplois d'utilité collective en garderie devrait être amorcée. Ce fonds servirait à la création de postes d'aides-éducatrices; 6) l'investissement requis (calculé au plein potentiel des places de gratuité [66 950] et d'exonération [129 530]) 
devrait être étalé sur cinq ou six ans. La première année, un montant d'environ 30 millions de dollars sera requis pour faire passer les enfants actuellement admissibles au nouveau régime d'exonération totale. Par la suite, on devrait consentir à développer entre 5000 et 6000 nouvelles places admissibles au régime par année ${ }^{12}$.

\section{Soutenir les parents dès la naissance... et avant}

\section{Offrir des visites à domicile}

Dans le but de soutenir le mieux possible les parents qui vivent des conditions économiques et familiales difficiles au moment de la naissance d'un enfant, les auteurs de Chacun sa part pensent que:

- des programmes de visites postnatales à domicile devraient être offerts sur tous les territoires de CLSC en s'appuyant sur les groupes ou services communautaires qui ont fait déjà la preuve de leur engagement dans ce domaine et en soutenant ces groupes de meilleure façon afin d'assurer leur stabilité et leur développement. Ces programmes devraient s'inspirer de stratégies qui ont déjà fait la preuve de leur efficacité auprès des familles à très bas revenus ${ }^{13}$.

\section{Assurer une meilleure préparation à la parentalité}

La maternité précoce conduit de nombreuses jeunes mères à l'aide de dernier recours et leurs enfants à la pauvreté. Cela ne représente ni une maladie ni une calamité; il est normal d'avoir des enfants quand on est adulte. Mais on peut s'inquiéter que tant d'enfants et de jeunes parents vivent dans l'insécurité, la précarité, la misère très souvent. Ces jeunes parents font partie d'une cohorte dans laquelle le taux de grossesse à l'adolescence a augmenté de $36 \%$ en l'espace de dix ans. Plusieurs jeunes filles et jeunes femmes empruntent la voie de la maternité pour arriver à l'autonomie. Les auteurs de Chacun sa part ne peuvent, à cet égard, que répéter ce que d'autres groupes ont affirmé avant eux et notamment les auteurs de la politique québécoise en périnatalité ${ }^{14}$. Ils invitent donc

- le ministère de la Santé et des Services sociaux à intensifier ses campagnes de prévention des grossesses à l'adolescence et les écoles secondaires à revoir et à renforcer leurs stratégies et leurs approches en matière d'éducation et de soutien à une parentalité et à une sexualité responsables. 


\section{Assurer une meilleure intégration à l'emploi}

pour les mères de jeunes enfants

Une majorité des enfants de 4 ans et moins inscrits à l'aide de dernier recours vivent dans des familles monoparentales féminines. De fait, $74 \%$ des familles monoparentales québécoises avec enfants de moins de 6 ans se retrouvent à l'aide sociale ${ }^{15}$ : il y en avait 97000 en janvier 1995. Sensibles aux perceptions et aux besoins des mères monoparentales pauvres et inscrites à l'aide sociale, les auteurs de Chacun sa part $\mathrm{n}^{\prime}$ en sont pas moins inquiets des effets négatifs que peut engendrer une fréquentation prolongée de l'aide sociale. Les meilleurs prédicteurs d'une trajectoire de pauvreté chronique pour ces mères sont: une sous-scolarisation, un stage prolongé de la mère à l'aide sociale dans ses premières années de vie parentale, une deuxième grossesse et l'absence de vie maritale durant ces premières années ${ }^{16}$. La politique actuelle des programmes $d^{\prime}$ aide consistant à octroyer un barème amélioré dit de non-disponibilité aux parents d'enfants de moins de 6 ans (1 200\$ par année) pourrait avoir pour effet $d^{\prime}$ 'isoler les mères $d^{\prime}$ 'un réseau de soutien indispensable à leur développement et à leur bien-être et d'inciter à la non-participation à des mesures de scolarisation et de préparation à l'emploi, mesures sans lesquelles ces mères augmentent les risques de se condamner à la pauvreté chronique, et avec elles leur enfant. Aussi, les signataires de Chacun sa part externe recommandent:

- de remplacer le barème de non-disponibilité s'étendant sur une période de 72 mois par d'autres modalités d'aide, notamment par un supplément pour nourrisson et par la gratuité des services de garde pour toutes les familles à faible revenu. Ce supplément pour nourrisson représente un montant de l'ordre de $100 \$$ par mois offert à compter du huitième mois de grossesse jusqu'au douzième mois suivant la naissance aux quelque 20000 nourrissons de familles à bas revenus recensés annuellement au Québec.

Cette disposition n'immunise pas les familles contre la pauvreté et ses effets. Elle vient toutefois donner un coup de pouce durant une période où les dépenses augmentent et où les parents ont besoin de toutes les ressources financières possibles sans lesquelles il leur est beaucoup plus difficile d'assurer un bon départ à l'enfant et à leur relation d'attachement avec l'enfant. Cette proposition a été amenée à la condition que la réforme offre aussi l'accessibilité gratuite aux services de garde pour les familles à bas revenus. Elle a aussi été avancée dans le contexte où les besoins des enfants sont désormais couverts par une allocation des enfants (voir point suivant). De même, cette proposition exige le développement de possibilités réelles pour 
les parents (les mères surtout) de se donner un plan d'insertion sociale et professionnelle et de le mettre en œuvre le plus rapidement possible. C'est pourquoi les auteurs jugent important: 1) d'apporter un soin particulier aux étapes d'orientation et de planification de leur insertion de façon à éviter à ces mères de longs et inutiles détours dans leur quête d'autonomie ; 2 ) d'abolir ce qui pourrait représenter de sérieux obstacles à leur démarche et aux solutions collectives qu'elles peuvent concevoir dont, explicitement, la clause de pénalité du partage de logement; 3 ) de soutenir ces mères dans tout effort qui viserait à les sortir de l'isolement et du sentiment d'accablement et d'impuissance qu'elles peuvent avoir acquis à l'égard de leur capacité à s'en sortir ${ }^{17}$; 4) d'adopter une approche plus familiale $q u^{\prime}$ individuelle dans le cadre d'un Plan de services familiaux. Ce plan devrait couvrir à la fois les besoins en formation ou en insertion de la mère, ceux concernant d'autres dimensions comme les loisirs, I'alimentation, le logement, le transport et ceux de son enfant; 5) d'orienter davantage les mères de familles monoparentales vers des programmes de scolarisation qui répondent à leur situation; 6) de maintenir, pour ce groupe de personnes, un accès privilégié à des programmes de retour aux études postsecondaires.

\section{Améliorer la situation économique des enfants}

en intégrant et redistribuant tous les revenus disponibles à leur égard dans une seule allocation

L'aide financière annuelle consentie aux enfants québécois dépasse largement les 3 milliards (ressources fédérales et québécoises confondues). Ces programmes visent à couvrir les besoins essentiels des enfants de familles à faible revenu, à préserver l'équité entre les familles qui ont des enfants et celles qui n'en ont pas (universalité de certains programmes) et à aider les parents qui ont de nombreux enfants. Pour arriver à maximiser l'impact de ces mesures, à les offrir également à toutes les familles à bas revenus (et à simplifier le système d'aide), les auteurs de Chacun sa part suggèrent:

- de créer une prestation unifiée pour les enfants (Allocation des enfants) qui prendrait l'allure d'une allocation mensuelle. Tout en conservant une composante universelle applicable à toutes les familles, cette allocation serait plus généreuse pour les familles démunies. Cette allocation unifiée est dessinée de manière à simplifier et unifier les ressources d'aide financière, à réduire la pauvreté, à faciliter la planification et les prévisions budgétaires pour les familles; 
- de fixer cette allocation, identique pour tous les enfants peu importe leur rang dans la famille, à un maximum de $3000 \$$, lequel comprendra une composante sélective $(2450 \$)$ et une composante universelle minimale ( $550 \$)$, la composante sélective commençant à diminuer progressivement à hauteur de $20000 \$$ de revenu familial jusqu'à un seuil de 60000 \$ à partir duquel seule la composante universelle s'appliquera.

Par ailleurs, devant la détresse économique de plusieurs milliers de familles monoparentales et considérant les conséquences néfastes que peut engendrer une fréquentation chronique de la pauvreté, le rapport propose de:

- maintenir un crédit d'impôt pour les familles monoparentales de l'ordre de $1300 \$$ par famille ${ }^{18}$.

\section{PrÉVENIR l'ENLISEMENT dANS la PAUVRETÉ}

\section{Reconnaître la situation des moins de 25 ans}

En juin 1995, 27326 jeunes adultes de moins de 21 ans (5\% des adultes présents à l'aide de dernier recours) et 47913 jeunes de 21 à 24 ans apparaissaient au fichier de l'aide de dernier recours. Un regard sur les vingt dernières années révèle que le taux $d^{\prime}$ inscription à l'aide sociale des $18-20$ ans est passé de $4 \%$ en 1975 à $10 \%$ en 1994. Le bond est encore plus important chez les 21-24 ans: le taux est passé de $4 \%$ à $13 \%$; durant les cinq dernières années, il a progressé de $2,9 \%$ chez les $18-20$ ans et de $5,3 \%$ chez les $21-24$ ans. Le taux des 21-24 ans n'a jamais été aussi élevé. De fait, le taux à l'aide de dernier recours augmente comparativement au taux $d^{\prime}$ inscription à I'assurance-chômage. Ainsi, en 1989, les taux d'inscription à l'assurance-chômage et à l'aide de dernier recours étaient de 9,9\% et de $6,4 \%$ respectivement; en 1994 , les taux basculaient à $6,8 \%$ et $11,6 \%$. Les périodes de récession font plus mal aux jeunes et les périodes de reprise leur profitent moins.

La faible scolarisation des moins de 25 ans inscrits à l'aide de dernier recours a de quoi inquiéter. Chez les moins de 21 ans, $78 \%$ déclarent moins de 11 ans de scolarité; chez les 21-25 ans, 69\%. Autre donnée préoccupante: $41 \%$ des inscrits se déclarent non participants aux mesures. Bien que ce taux soit inférieur aux $46 \%$ de non-participants chez les plus de 30 ans, il est accablant pour qui souhaite que ces jeunes adultes puissent atteindre l'autonomie financière le plus rapidement possible et occuper un emploi et un rôle social valorisé dans leurs communautés. L'examen de la génération 
des prestataires âgés de moins de 21 ans en 1983 montre que $41 \%$ sont toujours à I'aide sociale dix ans plus tard: $18 \%$ y sont demeurés en permanence (plus de 9000 jeunes), tandis que $33 \%$ (près de 18000 jeunes) y faisaient des allers et retours. D'autres données suscitent des inquiétudes quant à la reproduction intergénérationnelle de l'inscription à l'aide de dernier recours. Des 27576 jeunes de moins de 21 ans inscrits en 1993, 62\% venaient de familles dont les parents avaient eux-mêmes été à l'aide de dernier recours. Ces jeunes adultes sont plus faiblement scolarisés et ont vécu en plus grande proportion soit dans une famille monoparentale, soit dans une famille elle-même inscrite plus de cinq ans à l'aide de dernier recours ou soit dans une famille ou un centre d'accueil.

\section{Aider les jeunes: exprimer d'abord nos valeurs}

La façon dont une société $d^{\prime}$ adultes aide ses jeunes repose sur un ensemble de valeurs trop souvent implicites qu'il nous faut clarifier, ce que les auteurs de Chacun sa part se sont efforcés de faire en écoutant, en consultant, en lisant, en discutant avec les jeunes adultes et avec celles et ceux qui les côtoient. De ces consultations et réflexions, les auteurs concluent: 1 ) qu'il est illégitime que la société fasse "poireauter» ses jeunes inutilement, qu'elle économise sur leur dos, qu'elle fasse payer ses carences et ses erreurs par la génération qui arrive ; 2) qu'il est tout aussi illégitime que l'État se substitue aux parents et à la communauté dans leurs tâches de soutien aux jeunes ; 3) que la sécurité du revenu, bien qu'elle ne puisse être neutre dans ses effets, ne doit pas devenir une occasion de se bricoler un parcours tordu vers la vie adulte, parcours qui a toutes les chances de mener nulle part, sinon à la sécurité du revenu; 4) qu'il est normal qu'une société consente à donner un coup de pouce à ses jeunes, qu'elle leur fasse une place, qu'elle les prépare à une vie d'adulte responsable et compétent et qu'elle fasse sentir qu'elle a besoin de leur contribution; 5) qu'il est intolérable que l'on puisse accepter que des jeunes adultes ne soient nulle part et ne se dirigent nulle part; 6) qu'il serait irresponsable de leur accorder la sécurité du revenu en $n^{\prime}$ exigeant $d^{\prime}$ eux aucun effort d'apprentissage ou d'insertion.

\section{Offrir (enfin) une meilleure formation professionnelle}

La vie adulte implique I'autonomie financière; le jeune adulte s'y prépare. Les uns choisissent le parcours le plus long des études postsecondaires. Une autre partie des jeunes recherchent une voie plus courte. Dans ce dernier cas, ce sont très souvent des jeunes dont la 
curiosité, les centres d'intérêt et le talent nichent dans le concret, dans l'action, plutôt que dans les concepts et la théorie. À ceux-là, l'école $n^{\prime}$ a pas beaucoup à offrir : les programmes d'apprentissage et de formation professionnelle, lorsqu'ils sont disponibles, ne réussissent pas à les intéresser. Ces programmes sont rares, sous-financés, encore déconnectés des lieux de travail, ils arrivent tard dans le cheminement et sont éloignés des milieux de vie des jeunes. Avec nos maigres 5000 diplômes par année dans le secteur professionnel, nous sommes très loin du compte. La proportion des jeunes étudiants québécois de moins de 20 ans décrochant un premier diplôme au professionnel ou à titre d'apprenti est de $2,1 \%$; elle est de $68 \%$ en Allemagne, de $67 \%$ en Suisse, de $61 \%$ en Suède et elle se chiffre à $38 \%$ pour l'ensemble des pays de $\mathrm{I}^{\prime} \mathrm{OCDE}{ }^{20}$ ! II y a tout un rattrapage à faire.

Le Groupe de travail sur la relance de la formation professionnelle des jeunes au secondaire et de la formation technique (groupe Pagé) s'est longuement penché sur l'inefficacité du système d'enseignement professionnel dans nos écoles secondaires. Il conclut qu'il nous faut désormais consentir à des efforts considérables si l'on veut sérieusement infléchir la désaffection des jeunes vis-à-vis de la formation professionnelle. Les auteurs de Chacun sa part font leurs la philosophie, les approches et l'ensemble des recommandations du groupe Pagé. À ce titre, ils réitèrent la recommandation de:

- procéder dans les délais les plus brefs à une refonte complète du programme de formation professionnelle en milieu scolaire,

notamment dans le cadre des actions suivantes: 1) rapprocher l'enseignement professionnel des communautés et, à ce titre, revoir la carte des enseignements qui obligent des milliers de jeunes à quitter leur famille et leur région pour avoir accès à une formation appropriée; 2) amorcer, dès la première année du secondaire, un Plan individuel de formation où l'occasion serait donnée au jeune de $s^{\prime}$ informer des divers programmes, métiers et professions et de s'accrocher à un objectif réaliste et motivant; 3 ) offrir le plus tôt possible au secondaire la possibilité de s'inscrire au profil de formation professionnelle $\mathrm{e}^{21}$; 3) porter une attention particulière aux jeunes garçons dont le taux de décrochage est beaucoup plus élevé que celui des filles; 4) offrir une Attestation de formation professionnelle à celles et ceux qui mèneraient à terme une formation dans des métiers semi-spécialisés ; 5) informer les parents des possibilités de formation et $d^{\prime}$ emplois dans le secteur professionnel ${ }^{22}$;6) maintenir et enrichir le programme $d^{\prime}$ aide financière incitative pour les élèves du secondaire optant pour le programme de formation professionnelle. 
De plus, Chacun sa part reconnaît l'immense besoin d'ouvrir aux jeunes adultes des occasions d'apprendre leur métier dans un environnement de travail. II reconnaît aussi la situation familiale ou scolaire particulièrement pénible vécue par de nombreux jeunes antérieurement à leur inscription à l'aide. En ce sens, il recommande :

- d'accélérer le développement du régime d'apprentissage ${ }^{23}$ mis en œuvre sous le leadership de la SQDM en collaboration avec le ministère de l'Éducation et de fixer un objectif réaliste mais exigeant en la matière ;

- d'annuler le délai de 24 mois requis pour être admissible au rattrapage scolaire et de faire de l'accès à la Formation ${ }^{24}$ (dont la formation professionnelle) une priorité pour les jeunes admissibles à I'aide de dernier recours ;

- d'accepter (pour une période indéterminée) que des jeunes de 18-25 ans dont les parents auraient été inscrits durant plus de 5 ans à l'aide de dernier recours puissent s'inscrire dans les secteurs de formation professionnelle et recevoir une pleine prestation.

\section{Adopter une nouvelle approche d'accompagnement des jeunes}

Chacun sa part propose une nouvelle approche auprès des 18-25 ans suivant laquelle la prestation accordée aux jeunes adultes reposera sur une obligation réciproque jeunes-État dans le cadre d'une entente formelle qui définit un Plan d'insertion sociale et professionnelle. La réciprocité de l'engagement et de l'effort est indispensable à l'équité du système et à la réussite de cette nouvelle approche. D'une part, le jeune adulte entreprend ou termine une démarche d'insertion; d'autre part, l'État, ses ressources et les ressources de la communauté offrent les conditions nécessaires à la réalisation de cet engagement. Nous $\mathrm{n}^{\prime}$ insisterons jamais assez sur cet élément: en contrepartie de l'engagement des jeunes adultes dans un plan d'insertion, l'État s'oblige à offrir un parcours qualifiant à tout demandeur. Les signataires du rapport s'accordent pour affirmer que cette nouvelle approche de soutien financier aux jeunes adultes ne peut prendre place sans que les occasions et possibilités réelles de s'engager dans un Plan d'insertion professionnelle ou sociale qualifiant $n^{\prime}$ existent vraiment et qu'à ce titre le régime actuel devrait être maintenu aussi longtemps que les ressources humaines et communautaires d'insertion ne seront pas disponibles pour accueillir les jeunes adultes dans une diversité de parcours capables de les mener à des objectifs valorisants ${ }^{25}$. 
Il est extrêmement important de donner clairement le signal à l'ensemble de la communauté que l'approche avec les jeunes ne doit pas en être une de "serrage d'ouïes" ou de "serrage de vis»! Nos propositions reposent sur la confiance d'une société envers ses jeunes et non sur la méfiance. Elles veulent donner un élan nouveau aux jeunes, même à ceux à propos desquels ont aurait envie de dire qu'il $n^{\prime} y$ a rien d'autre à faire que de les laisser se débrouiller. Suivant ces mises en garde, les signataires de Chacun sa part recommandent:

- de conserver le montant de la prestation des jeunes à parité avec les 25 ans et plus (6000\$). Il faut rappeler ici que ce montant est assujetti à la clause de la contribution parentale et au statut de vie maritale. Il faut également rappeler que les prestations québécoises d'aide sociale ont été dessinées pour couvrir les besoins de subsistance (besoins essentiels) à partir des « dépenses essentielles effectuées par les $10 \%$ des ménages de travailleurs et travailleuses les plus pauvres ${ }^{26} »$;

- la mise à condition d'une partie de la prestation $(2000 \$)^{27}$ contre participation à un Plan d'insertion sociale et professionnelle. Cette disposition est sans doute difficile à accepter pour plusieurs citoyens, mais elle s'impose dans la mesure où les données indiquent l'urgence de prendre les moyens pour prévenir l'installation de vies à l'aide sociale. Il faut cependant user avec discernement et respect de cette règle de l'aide conditionnelle.

Dans ce contexte, il devient extrêmement important que:

- le suivi du PISP se fasse selon un mode d'échange d'informations entre les jeunes, les organismes promoteurs ou de formation et les conseillers à l'emploi ${ }^{28}$;

- tous les acteurs collaborent à la mise en œuvre de ressources communautaires pour jeunes capables de répondre à l'ensemble de leurs besoins et de mettre à profit leurs énergies et leur créativité.

\section{Créer des emplois pour les jeunes adultes}

Autant faut-il se résoudre à prévenir le fait que des jeunes adultes se retrouvent sur la touche à 18 ans, autant faut-il s'acharner à ouvrir les perspectives d'emploi. Le taux de chômage atteint $20 \%$ chez les $15-19$ ans et $17 \%$ chez les $20-24$ ans, alors qu'il est de $12 \%$ chez les plus de 25 ans. II a bondi de 4 points en quatre ans chez les 20-24 ans. En période de crise, les jeunes adultes éprouvent plus de difficultés à demeurer sur le marché du travail et à se qualifier pour 
I'assurance-chômage en cas de coup dur. Ainsi, lors de la récession de 1982, le taux de chômage des moins de 25 ans atteignait $23 \%$ contre $13 \%$ chez les plus de 25 ans; il tombait à $13 \%$ en 1989 (8\% chez les 25 et plus) et remontait à $18 \%$ en 1992, un écart de 6,5\% avec celui des plus de 25 ans $^{29}$. La prochaine décennie sera celle d'un effort particulier pour faire une place aux jeunes adultes sur le marché de I'emploi. Les signataires de Chacun sa part recommandent au gouvernement :

- d'adopter une politique de l'emploi qui favorise l'embauche des jeunes.

Cette politique pourra envisager les éléments suivants: 1 ) réduire le temps de travail ; 2 ) ouvrir le débat sur la question de l'âge de la retraite ; 3 ) interdire la réembauche des retraités dans la fonction publique et dans les entreprises privées ; 4) développer dans toutes les régions du Québec des emplois d'utilité collective capables de multiplier, pour les jeunes adultes, les occasions de se trouver un premier emploi valorisant dans leur propre communauté; 5) offrir aux employeurs (de corporations à but lucratif ou sans but lucratif) une exonération des charges sociales pour les trois premières années du premier emploi d'un jeune adulte de moins de 25 ans. Cela pourrait $\mathrm{s}^{\prime}$ appliquer dans un premier temps, à titre expérimental, aux parents de jeunes enfants.

De plus, nous pensons qu'il faudrait éventuellement, après rodage de la nouvelle formule:

- offrir le plus rapidement possible le programme APPORT (ou ce qui en tiendra lieu; voir dernière section) aux jeunes adultes sans enfants de 22-24 ans dans le but de les encourager à se maintenir en emploi et d'éviter qu'ils ne fassent l'expérience d'une trop grande pauvreté.

\section{PrÉVENIR LA MISE À l'ÉCART DES GROUPES PARTICULIERS}

\section{Inclure les personnes présentant des contraintes sévères}

Parmi les 47000 participants aux mesures d'insertion en emploi offertes aux personnes inscrites à l'aide de dernier recours, on en compte seulement $5 \%$ ou $6 \%$ qui présentent des contraintes sévères, alors que ces personnes représentent dans les $20 \%$ des adultes inscrits. Outre la présence même de contraintes très sérieuses chez une grande partie de ces personnes, il y aurait plusieurs causes à la rareté de la participation des personnes présentant des contraintes 
sévères. Premièrement, le système aurait tendance à tenir pour acquis que le statut $d^{\prime}$ " inapte» au travail définit une quasi-impossibilité $d^{\prime}$ intégration pour ces personnes. Deuxièmement, la présence d'un barème plus élevé induirait une urgence moins grande $d^{\prime}$ offrir des services d'intégration à l'emploi à ces personnes. Troisièmement, dans un contexte de surcharge et d'isolement, le degré de difficulté éprouvé par les agents avec ce groupe de personnes pourrait les pousser à considérer d'abord les autres dossiers. Quatrièmement, les entrepreneurs et employeurs pourraient être réfractaires à se compromettre dans le cas de personnes réputées moins compétitives que $d^{\prime}$ 'autres. Cinquièmement, les personnes inscrites au programme ne seraient pas suffisamment informées de leur droit à s'insérer dans une mesure, ou même de leur droit à un revenu de travail exempté de réductions de leur prestation.

Une telle situation engendre chez ces personnes la pauvreté et I'exclusion chroniques; aussi Chacun sa part recommande-t-il, parmi un train important de mesures, de:

- conserver avec ces personnes un objectif d'intégration sociale et professionnelle dans le contexte d'une démarche évolutive où le critère de succès reposera davantage sur un processus $d^{\prime}$ acquis que sur des statistiques de sortie du système ;

- offrir aux personnes présentant des contraintes sévères à l'emploi autre chose qu'un diagnostic qui confirme leurs incapacités. À cet égard, il y a besoin de modifier considérablement les formulaires de prise de connaissance de ces personnes de façon qu'elles puissent aussi faire état des talents, ressources, intérêts et ambitions qu'elles peuvent se reconnaître;

- identifier et soutenir financièrement les entreprises et groupes communautaires $d^{\prime}$ insertion au travail capables de mettre à profit les atouts, les centres d'intérêt, les aspects positifs, les habiletés, les forces des personnes dans une approche de développement plutôt que dans une approche de thérapie occupationnelle;

- élargir le programme de subventions compensatoires ou de prêts garantis aux entreprises qui engagent les chômeurs de longue durée et les personnes présentant des contraintes sévères à l'emploi ;

- renforcer le programme de Centres de travail adapté (CTA) de I'OPHQ. Ces centres emploient 1700 personnes, dont $80 \%$ sont inscrites à l'aide de dernier recours. 


\section{Assurer l'insertion sociale des personnes présentant des problèmes psychosociaux}

Outre les personnes souffrant de contraintes sévères, on estime aux environs de 100000 le nombre de personnes qui présentent des caractéristiques personnelles ou socioprofessionnelles qui les rendent peu ou pas attrayantes pour les employeurs. Ce sont des personnes qui peuvent éprouver des problèmes importants de motivation, de manque de confiance en soi, des symptômes de dépression ainsi que des problèmes de communication, d'isolement social, de toxicomanie, des formes de psychoses passagères, de déficience intellectuelle légère ou moyenne. Ces personnes ne doivent pas être laissées pour compte dans une approche où l'insertion, l'emploi et la sortie rapide de I'aide deviendraient l'unique obsession. Une étude évaluative du MSR indique qu'une approche tenace et personnalisée permet à un plus grand nombre de ces personnes de passer à des mesures actives $\mathrm{d}^{\prime} \mathrm{emploi}^{30}$. Aussi les signataires de Chacun sa part recommandent-ils fortement au ministère de l'Emploi et de la Solidarité de:

- inscrire très fermement dans sa mission le développement et le maintien de programmes de pré-emploi (Insertion) capables d'accueillir et de soutenir l'insertion sociale et économique des personnes présentant des problèmes d'adaptation psychosociale ;

- suivre l'évolution de la part relative des Plans d'insertion sociale et professionnelle élaborés avec ce groupe de personnes en visant une augmentation régulière de cette part ;

- $\quad$ 'associer, dans la planification et dans la mise en œuvre de ces programmes, des intervenants des communautés qui ont une longue expérience de l'accompagnement de ces personnes. On pense notamment ici aux SEMO et aux entreprises d'insertion à I'emploi ;

- établir des protocoles de reconnaissance des acquis (à la manière d'un CV) pour ces personnes de sorte que les heures travaillées et rémunérées et les heures de participation non rémunérées puissent être reconnues et inscrites dans le cadre d'un parcours vers l'emploi ;

- établir clairement avec les CLE la distinction entre heures travaillées et bénévolat. Les heures travaillées doivent être rémunérées et soumises aux normes minimales du travail ;

- élaborer des protocoles simples de collaboration qui favoriseraient la mise en contact des usagers du système de santé (CLSC) 
avec les services des CLE, notamment en ce qui a trait aux programmes Insertion et Formation.

\section{Reconnaître la situation particulière des plus âgés}

La perte d'un emploi représente un obstacle à l'intégration professionnelle majeure pour les 55 ans et plus. La loi actuelle le reconnaît en octroyant aux personnes de 55 ans et plus inscrites à l'aide sociale un supplément de barème dit de non-disponibilité aux programmes d'employabilité. Cependant, ces personnes sont soumises au test $d^{\prime}$ actif très exigeant introduit à la faveur de la loi 115 en ce qui a trait à leur admissibilité à l'aide, ce qui, à toutes fins utiles, les oblige à liquider le patrimoine qu'elles auraient pu accumuler durant leur vie active. Ainsi, Chacun sa part recommande de:

- permettre aux personnes de 55 ans et plus de protéger leurs acquis au moyen d'un test d'actif plus généreux en renonçant au supplément pour non-disponibilité et de financer cet élargissement du test d'actif à même les économies de renonciation au supplément.

\section{Améliorer le sort des personnes immigrantes}

De 9000 ménages en 1975, la population immigrante inscrite à l'aide de dernier recours est passée à 63000 ménages en 1994, une augmentation de $600 \%$. Cette augmentation se compare à une hausse de $140 \%$ dans I'ensemble des ménages. L'ensemble des ménages nés à l'extérieur du Canada représente désormais $13 \%$ des inscriptions à I'aide de dernier recours comparativement à $4,5 \%$ en 1975 . Le taux des personnes présentant des expériences de travail est plus bas dans cette population ( $53 \%$ ) que dans I'ensemble des aptes au travail $(66 \%)$. Cela est en grande partie dû à une présence féminine moins grande sur le marché de l'emploi parmi les ménages immigrants. Autre caractéristique extrêmement importante: la sur-représentation des emplois à statut professionnel parmi les adultes immigrants inscrits à l'aide sociale.

La plupart des problèmes relevés à propos des personnes immigrantes inscrites à l'aide de dernier recours ne sont pas très différents des problèmes de l'ensemble des personnes inscrites. Toutefois, dans le but de faciliter l'insertion sociale et économique des personnes immigrantes qui s'adressent à la sécurité du revenu, les signataires de Chacun sa part recommandent notamment que: 
- les services à ce groupe soient offerts dans des CLE spécialisés afin $d^{\prime} y$ concentrer toutes les ressources linguistiques et culturelles nécessaires à la livraison de services d'ajustement de revenus et de services-conseils;

- les CLE spécialisés auprès des personnes immigrantes établissent des liens de partenariat privilégiés avec ces groupes communautaires de façon à favoriser la définition de besoins spécifiques et prioritaires et $d^{\prime} y$ répondre adéquatement;

- les CLE élaborent en collaboration avec les acteurs sociaux et économiques locaux des stratégies de création d'emplois d'utilité collective appropriées aux personnes immigrantes, notamment dans la gamme des services d'accueil et d'accompagnement des nouveaux arrivants, de leur orientation professionnelle et de leur intégration à la communauté sociale et économique francophone, des loisirs pour les jeunes, des interventions sociosanitaires, de I'aide aux parents de jeunes enfants et aux parents d'adolescents, de l'aide aux enseignants dans les écoles ou dans tout autre domaine jugé pertinent ;

- un programme de Retour aux études postsecondaires pour personne immigrante diplômée soit offert pour permettre la mise à niveau des personnes qui auraient terminé des cycles d'études collégiales ou universitaires à l'étranger ;

- le Conseil du patronat, les syndicats et les associations à vocation commerciale et manufacturière soient mis à contribution dans la tenue d'une étude et dans la mise sur pied d'un programme visant à définir les obstacles et les stratégies à l'insertion des personnes immigrantes dans la vie économique en région.

\section{PRÉVENIR L'ÉROSION DE LA SOLIDARITÉ}

\section{Couvrir le coût des médicaments de tous les pauvres}

Au moment d'écrire Chacun sa part, les prescriptions médicales étaient totalement couvertes pour les personnes inscrites à l'aide sociale à partir d'un carnet de réclamation et d'une liste de médicaments homologués. Cette couverture était toutefois limitée aux seuls prestataires de la sécurité du revenu, alors que le besoin existe aussi chez les travailleurs et travailleuses à faible revenu. Cette situation inéquitable a été très souvent évoquée lors de nos consultations comme un motif de ressentiment, une menace à la solidarité chez les groupes de citoyens à bas revenus et comme un frein à l'emploi 
en particulier pour les personnes qui devraient alors payer des coûts élevés pour elles ou leur famille. Les signataires ont alors recommandé:

- d'étendre la protection accordée aux prestataires pour leurs besoins de santé à la catégorie suivante des travailleurs au bas de l'échelle, de manière à éviter cette distorsion dans les choix économiques des personnes ;

- de mettre sur pied, pour ce faire, un régime de co-assurance dans lequel la franchise qui pourrait être imposée serait limitée à $120 \$$ pour les personnes à faible revenu, et que ce montant soit fourni aux personnes admissibles à une prestation de sécurité du revenu. Le niveau de co-assurance (la contribution de l'usager au coût de chaque médicament au-delà de 120 \$) pour ces personnes ne devrait pas dépasser $20 \%$, jusqu'à un maximum de $176 \$$ par personne pour les premiers $1000 \$$ de dépenses de médicaments, le reste étant gratuit;

- d'offrir la couverture gratuite aux enfants de 0 à 17 ans et aux personnes présentant des contraintes sévères à l'emploi ${ }^{31}$.

\section{Définir le logement comme espace de solidarité}

Le régime d'aide de dernier recours prévu par la loi 37 a maintenu le revenu des personnes prestataires de la sécurité du revenu sur la limite la plus minimale possible de la subsistance, récupérant ainsi toute économie d'échelle qui pouvait être réalisée par des comportements de débrouillardise. Cette attitude a donné lieu à deux dispositions particulièrement décriées pour leur caractère désincitatif à la solidarité et pour leur négation du droit des personnes à faire des choix économiques: 1) la coupure des prestations pour partage du logement, laquelle a pour effet de retirer $104 \$$ du chèque mensuel d'aide sociale des personnes partageant un même logement; 2) I'application d'un test de logement qui diminue d'autant les prestations quand le coût du loyer s'avère plus bas qu'un seuil déterminé. Parallèlement, une troisième mesure a été établie pour répondre aux besoins particuliers des ménages avec enfants: une allocation au logement d'un maximum de $90 \$$ par mois pour les familles dont le fardeau locatif est trop élevé comparativement à leur revenu.

Dans un système de sécurité du revenu fiscalisé et complémentaire à des revenus d'emploi, tel que préconisé par les auteurs de Chacun sa part, on peut remettre en question un processus faisant intrusion dans les choix budgétaires des personnes. Dans un tel système, l'allocation minimum prévue au titre de la sécurité du revenu 
doit être considérée comme un revenu de base permettant la survie pour l'ensemble des besoins du ménage, incluant le logement. Par ailleurs, les auteurs sont d'avis qu'il vaut mieux confier l'ensemble du processus de couverture du logement aux instances dont $c^{\prime}$ est la principale mission. C'est pourquoi le rapport recommande:

- I'abolition de la coupure pour partage du logement et l'abolition du test de logement, deux mesures incompatibles avec l'esprit d'un régime de sécurité du revenu intégré à la fiscalité et qui ne sauraient être considérées comme des économies justifiées;

- I'intégration de l'ensemble des politiques d'aide au logement, y compris l'allocation au logement actuellement administrée par la Sécurité du revenu, dans une politique intégrée du logement social qui serait administrée par la Société d'habitation du Québec ${ }^{32}$.

\section{Intégrer progressivement la prestation de base à la fiscalité}

En 1970, le Québec instaurait un filet de sécurité destiné à assurer un revenu de subsistance à l'ensemble des Québécois et Québécoises. Cette loi d'aide de dernier recours n'a cependant pas réglé la question de l'intégration des personnes appauvries au marché du travail. Au contraire, elle a amené les gens à percevoir la prestation de base comme un revenu principal calculé indépendamment des revenus de travail, où ceux-ci deviennent peu désirables puisqu'ils réduisent d'autant la prestation de base. C'est pourquoi l'on a instauré en 1979 un programme de supplément aux revenus de travail, le programme SUPRET, premier du genre au Canada. Avec ce programme, les familles à faible revenu pouvaient compléter leur revenu de travail par une aide complémentaire, à mi-chemin entre les revenus gagnés et un seuil de faible revenu. Toutefois, l'évaluation des revenus étant faite à partir des revenus de I'année précédente, le programme a connu des problèmes d'ajustement qui l'ont rendu peu populaire et ont conduit à son remplacement. Une nouvelle étape a été franchie avec l'instauration du programme APPORT (Aide aux parents pour les revenus de travail). Une innovation majeure d'APPORT par rapport à SUPRET a été de considérer le supplément accordé comme un ajustement au revenu de l'année en cours. Cela demande certes des ajustements supplémentaires et une certaine ingéniosité dans le calcul des mensualités, mais permet d'offrir le soutien au moment où il est nécessaire. On a aussi résolu l'harmonisation avec la fiscalité en fixant la fin du supplément au seuil de revenu imposable, ce qui évite des chevauchements inutiles. 
On voit à travers ce tableau se dessiner très nettement l'enjeu $d^{\prime}$ un traitement le plus universel possible des besoins en soutien au revenu, à travers une administration fiscale unique qui prendrait autant en charge la partie accordée à ceux qui ne gagnent pas assez (fiscalité négative) que la partie perçue de ceux qui gagnent suffisamment pour partager et contribuer aux finances publiques (fiscalité positive). Il y a d'énormes avantages à tirer d'une intégration graduelle de la sécurité du revenu à la fiscalité: 1) on répond à une aspiration de tous, fortunés ou non, d'être traités sur un pied d'égalité. On remplace, au moins pour une partie, le statut d'assisté social par celui de contribuable ; 2 ) en unifiant le système de sécurité du revenu, on peut aussi appliquer le concept de façon plus large. L'État peut alors mieux jouer son rôle redistributeur en fonction de l'évolution des besoins, en tenant compte de l'ensemble des programmes de sécurité du revenu existants, et non seulement du programme d'aide de dernier recours ; 3) une approche plus intégrée à l'appareil du Revenu permet une économie de gestion où l'intégration de tous les programmes dans une logique fiscale par laquelle l'information de base provient de la déclaration de revenus complétée des informations propres aux programmes eux-mêmes permet une gestion plus simple et plus efficace ; 4) il est reconnu que le ministère du Revenu impose par sa capacité de vérification une "crainte salutaire». En intégrant le personnel de vérification au ministère du Revenu et en exerçant une vigilance tant sur la fiscalité positive que négative, on rétablit aussi la condition d'équité préalable à toute activité de contrôle bien acceptée. C'est pourquoi les auteurs de Chacun sa part ont opté pour les recommandations suivantes:

- maintenir le programme APPORT, dans une forme modifiée conséquente à l'instauration d'une allocation des enfants et portant le nom de PART (Prestation ajustée aux revenus de travail) pour toutes les familles actuellement admissibles à un supplément de revenus de travail ${ }^{33}$ et non pas pour les seules familles inscrites à l'aide sociale ;

- établir comme principe de calcul de la PART le seuil de revenu de base disponible au niveau du barème de non-participation actuel, soit 6000 \$, éliminer la notion de gains de travail exclus et supplémenter les revenus de travail entre $0 \$$ et le seuil de revenu imposable de sorte qu'à $0 \$$ de revenus de travail la PART soit de 6000 \$ et qu'elle diminue progressivement pour $s^{\prime}$ annuler au seuil de revenu imposable. Cela implique que pour chaque dollar gagné sur le marché du travail une partie de la PART est retenue, ce qui n'est pas le cas dans le présent système 
où pour chaque dollar gagné on soustrait un dollar de l'aide de dernier recours.

- instaurer en séquence le programme de Prestation ajustée aux revenus de travail (PART), d'abord auprès des familles avec enfants (ainsi que la situation actuelle le fait) et ensuite auprès des jeunes de 22 à 24 ans que l'on doit particulièrement soutenir dans leurs efforts $d^{\prime}$ acquérir l'autonomie financière ${ }^{34}$;

- évaluer rigoureusement chaque étape rapprochant le système de sécurité à la fiscalité avant d'étendre ce système à l'ensemble des personnes à bas revenus étant donné les enjeux économiques et sociaux en cause.

\section{Réformer la fiscalité}

II est impensable de songer à assurer une sécurité pour tout le monde et à réduire des iniquités réelles et lourdement ressenties, notamment par les travailleurs et travailleuses à faible revenu, sans opérer des déplacements significatifs dans I'ensemble de l'appareil fiscal. Une réforme de la fiscalité est nécessaire. L'obstination à vouloir réformer la sécurité du revenu à partir des budgets continuellement amputés de ce seul programme conduit inévitablement à la catastrophe, c'està-dire aux coupures de l'aide et à l'appauvrissement des plus pauvres ${ }^{35}$. Une meilleure progressivité de notre système fiscal contribuerait à rétablir la confiance de tous les contribuables, surtout celle des petits et moyens salariés qui ressentent plus que toute autre catégorie de citoyens le poids du fardeau fiscal, dans le système de contribution à l'avoir commun et de redistribution des revenus. Plusieurs postes fiscaux pourraient être examinés et réinvestis dans la réalisation de l'équité meilleure que suppose la réforme proposée. À titre indicatif et sans nous réclamer d'une étude en profondeur de ces postes qui nous permettrait de faire des recommandations précises, nous en proposons une liste: 1) les différences d'épargne sur le REER en faveur des contribuables à revenus élevés; 2) les reports d'imposition sur les intérêts des REER et sur les Régimes de pension accrédités (RPA), notamment en ce qui a trait aux investissements à l'étranger; 3) les reports d'impôts de grandes entreprises ou de grandes sociétés ; 4) la non-imposition des indemnisations d'accidents du travail ; 5) la non-imposition de certains gains en capital ; 6) la non-imposition des loteries et jeux; 7) les cotisations syndicales et professionnelles; 8 ) la déduction des frais financiers et d'intérêts ; 8) la non-taxation des frais de transactions boursières; 9) l'exonération des revenus des fiducies familiales. 


\section{CONCLUSION}

L'heure est au redressement des finances publiques. Le contexte politique actuel est lourdement chargé par la question de la réduction du déficit. Avant que l'élimination du déficit ne vienne hanter la nouvelle administration du présent gouvernement, la précédente avait entrepris trois chantiers majeurs: la réforme de la santé, celle de l'éducation et celle de la sécurité du revenu. Dans le cadre de cette dernière réforme, le mandat dont étaient chargés les membres du Comité externe stipulait notamment qu'elle devait contribuer à diminuer la pauvreté en respectant les contraintes budgétaires du gouvernement. Cette double exigence installait une tension importante dans les travaux du comité externe chargé de proposer un énoncé de réforme. Cette tension allait s'amplifier à l'arrivée de la nouvelle administration et ultimement contribuer (en partie) à la publication d'un rapport majoritaire et $d^{\prime}$ un rapport minoritaire. Le premier réduisait au minimum les coûts de la réforme, mais refusait d'abandonner la lutte à la pauvreté comme objectif prioritaire, alors que le second optait pour une proposition davantage alignée sur le redressement des finances publiques.

Pour les signataires du rapport majoritaire (Chacun sa part), le redressement des finances publiques ne peut reposer sur la base unique des réductions des dépenses, notamment sur la réduction des dépenses sociales consacrées aux sans-emploi. On ne peut demander aux personnes les plus pauvres de faire seules les frais d'une réforme et encore moins d'un redressement des finances publiques. II y a là des enjeux de solidarité, d'équité et, ultimement, de démocratie.

Ce qui est plus fondamentalement en cause dans la question de l'aide sociale, c'est la capacité de milliers d'adultes et d'enfants de se développer dans un contexte où les possibilités de se nourrir et de se loger convenablement, d'apprendre, de réapprendre et de s'affirmer comme des citoyens à part entière (dont on pourrait dire qu'ils partagent un droit fondamental: celui de rêver et d'espérer) dépendent non pas exclusivement de la motivation des individus, mais aussi, et surtout, du fait d'une reconnaissance collective et sociale du problème de la pauvreté et de ses effets. C'est à ce niveau que les positions se polarisent autour des deux rapports préparés par les membres du Comité externe sur la sécurité du revenu (Noël, 1996) ${ }^{36}$. L'un, minoritaire, entérine la logique du présent régime de la loi 37 , une logique individuelle, blâmante, autoritaire, qui remet à la personne I'obligation de se montrer et de se rendre employable dans le cadre d'un contrat individuel prescrit par l'État. L'autre, majoritaire, rejette cette approche, tente de remettre la personne au centre du système et de l'appuyer dans une logique de responsabilité collective qui 
reconnaît simultanément le droit aux individus de se concerter et de s'associer devant le pouvoir de l'État et le devoir de s'impliquer dans une démarche d'insertion. Le débat autour de l'aide sociale, $s^{\prime}$ il prend parfois l'allure d'un débat sur les finances publiques, est $\mathrm{d}^{\prime}$ abord un " débat autour d'une lente construction de la démocratie et de la citoyenneté »(Noël, 1996). La prévention de la pauvreté passe par une meilleure redistribution des revenus et par la création d'emplois, notamment au niveau local. Elle passe aussi par un enrichissement du rôle de citoyen et par une participation plus grande à l'exercice individuel et collectif de la démocratie.

\section{Notes}

1. L'auteur a été coprésident du Comité externe de la réforme de la sécurité du revenu. II a signé avec ses collègues Vivian Labrie et Alain Noël le rapport Chacun sa part. Le présent article est largement le résultat d'emprunts à la version originale du rapport. À ce titre, la contribution de Vivian Labrie et d'Alain Noël est très importante. La sélection et la structuration du matériel, de même que les modifications au texte original, sont cependant de la responsabilité exclusive de l'auteur. On pourra consulter la version originale du rapport : C. Bouchard, V. Labrie et A. Noël (1996). Chacun sa part. Ministère de l'Emploi et de la Solidarité: Gouvernement du Québec, 235 pages.

2. Voir, à ce sujet, Serge Paugam (1993). "Entre l'emploi et l'assistance: réflexion sur l'insertion professionnelle des allocataires du RMI ", Travail et emploi, 55, p. 81; et Isabelle Astier (1995). "Du récit privé au récit civil: la construction d'une nouvelle dignité ? ", Lien social et politiques, 34, automne: 121-130.

3. On voudra bien consulter les pages 93 à 114 du rapport pour une description détaillée de ces programmes.

4. Le nouveau réseau pourrait s'ajuster selon les cas aux territoires des MRC, municipalités et arrondissements urbains ou regroupements reconnus de quartiers dans les grandes villes, et comporter selon les cas des services communs ou des succursales.

5. Cette proposition de la création de Comité d'usagers, de même que celle voulant que les membres des Conseils de partenaires soient élus et non pas nommés, fait partie des divergences qui se sont manifestées entre les membres du Comité externe de la réforme de la sécurité du revenu. Les signataires de Chacun sa part attribuent une importance extrême à la démocratisation du système, la lutte à la pauvreté, passant aussi par la lutte à l'exclusion sociale et politique. Les membres du rapport minoritaire y voient plutôt des occasions de conflit.

6. Pierre Paquette (1995). Un Québec pour l'emploi. Montréal : Éditions Saint-Martin, p. 122-123.

7. Voir à ce sujet F. Aubry et J. Charest (1995). Développer l'économie solidaire: éléments d'orientation, p. 31.

8. Voir à ce sujet Le rapport du Comité d'orientation et de concertation sur l'économie sociale, publié en mai 1996, Gouvernement du Québec, 89 pages.

9. E. Young (1995). Investing in young children. World Bank discussion papers. Washington, DC.

10. Y. Le Bossé (1995). Impact des programmes d'employabilité sur le développement optimal des familles et des enfants: Analyse des expériences américaines. Charny: Argos Consultants. 
11. De très nombreuses recommandations de Chacun sa part visent à réduire les écarts entre l'aide offerte aux travailleurs à bas revenus et celle offerte aux personnes inscrites à l'aide sociale. Cela rejoint une préoccupation d'assurer une plus grande équité entre les groupes sociaux, de renforcer ainsi une solidarité grandement menacée et du même coup d'encourager l'effort.

12. Dans le but $d^{\prime}$ arriver à financer adéquatement cet effort de développement des garderies, le crédit d'impôt pour frais de garde ne devrait être maintenu que pour les frais reconnus par un service de garde régi (OSBL, garderies privées accréditées, agences de garderies familiales accréditées). Les économies ainsi réalisées devraient être réservées au développement de nouvelles places et à la consolidation du programme de gratuité et d'exonération. Le crédit d'impôt actuel consenti aux frais de garde sans égard à la nature des services reçus encourage le travail au noir, ralentit le développement d'un réseau de garde de qualité et encourage probablement les déclarations frauduleuses. Par ailleurs, les déductions fiscales pour frais de garde consenties par les gouvernements aux familles à revenus supérieurs pourraient être revues dans le but d'assurer une meilleure équité entre familles à revenu moyen et à revenu supérieur.

13. Voir à ce sujet C. Bouchard (1989), "Lutter contre la pauvreté ou ses effets? Les programmes d'intervention précoce ", Santé mentale au Québec, 14 : 138-149.

14. Gouvernement du Québec (1993). Politique de périnatalité. Québec: ministère de la Santé et des Services sociaux.

15. P. Lanctôt et Rousseau (1995). Profil des familles monoparentales, aptes au travail à I'aide de dernier recours. Profil $n^{\circ} 5$. Québec: ministère de la Sécurité du revenu.

16. F.F. Furstenberg, J. Brooks-Gunn et S.P. Morgan (1987). Adolescent Mothers in Later Life. New York: Cambridge University Press.

17. En particulier, les encourager à s'inscrire dans toute forme de projet associatif (ACEF, cuisines collectives, clubs d'emplois, coopératives de logement, regroupements de mères de familles monoparentales, etc.) qui viserait à la fois la sociabilité et l'amélioration de leur condition économique.

18. Toutes ces mesures de réaménagement constituent un effort de redistribution au regard des politiques de transfert des revenus envers les familles les moins fortunées.

19. On ne peut à partir de ces seules données faire état de I'ampleur du phénomène de transmission intergénérationnelle de l'inscription à l'aide sociale. Il faudrait pour cela disposer d'études indiquant combien parmi les enfants de familles inscrites à l'aide de dernier recours y sont eux-mêmes présents dix ou vingt ans plus tard. De plus, les règles d'assujettissement de l'aide à la contribution des parents écartent davantage les jeunes des familles moins en détresse. Cependant, ces données sont suffisamment sérieuses pour qu'on se préoccupe en particulier du sort des adultes les plus jeunes.

20. Groupe de travail sur la relance de la formation professionnelle des jeunes au secondaire et de la formation technique (1995). La formation professionnelle chez les jeunes: un défi à relever (Groupe Pagé). Québec: ministère de l'Éducation.

21. Le groupe Pagé recommande que ce soit dès après la troisième année du secondaire, mais on pourrait envisager une amorce sérieuse dès la mi-troisième de façon à inciter les élèves à demeurer dans le système scolaire.

22. Cette information auprès des parents d'élèves de $1^{\text {re à }} 5^{\mathrm{e}}$ secondaire pourrait être confiée à des conseillers recrutés dans le cadre du développement des emplois d'utilité collective.

23. Selon les données de la SQDM, un tel système d'enseignement dual (en entreprise et à l'école) réduit considérablement les écarts de chômage entre les jeunes et les autres groupes d'âge. Durant I'apprentissage auprès d'un compagnon, I'apprenti cumule deux statuts : celui de salarié et celui d'élève. Le système est reconnu par le MEQ. Le potentiel de développement est énorme, tant par le nombre d'élèves que par le nombre de métiers (169) auxquels il pourrait s'appliquer. En Allemagne, 500000 entreprises accueillent des apprentis; plus de la moitié de ces entreprises comptent moins de 50 employés (Klaus Rachwalsky [1991]. Le système dual de formation professionnelle en République fédérale allemande. Cologne: Carl Duisberg Gesellschaft). 
24. Chacun sa part propose une refonte des programmes de parcours vers l'emploi en quatre volets intégrés: Orientation, Insertion, Formation et Emploi. C'est au volet Formation que cette recommandation fait référence.

25. Cette condition et les nombreuses mises en garde du rapport ne sont pas inutiles. L'exemple américain à cet égard est à proscrire. Le président Clinton annonçait d'abord une réforme où les années consécutives de prestations sont réduites à deux et à cinq pour toute une vie. II conviait ensuite les entreprises américaines à créer de l'emploi pour ces personnes. Il est tout à fait évident que cela fait porter le risque de la réforme uniquement par les sans-emploi.

26. Comité sur la lutte à la pauvreté, La pauvreté au Québec: État de situation, problématique et exemple d'interventions possibles de lutte à la pauvreté. Québec: ministère de la Sécurité du revenu, septembre 1994, p. 12.

27. Dans le cas où un jeune refuserait de définir et de s'inscrire dans un PISP, il est dûment informé qu'il ne pourrait alors toucher que $4000 \$$ des $6000 \$$ de revenu de subsistance. Le jeune adulte est alors placé devant un choix. Les auteurs de Chacun sa part désapprouvent la proposition d'expulsion pure et simple telle que suggérée par les auteurs du rapport minoritaire.

28. On pourrait, par exemple, prévoir quatre rencontres annuelles entre les conseillers et les jeunes adultes, dont une avec la personne de la ressource de formation ou $d^{\prime}$ insertion. Ces rencontres se tiennent dans le double objectif de s'assurer de la pertinence du parcours inscrit au PISP pour le jeune et du respect du PISP par toutes les parties. À sa demande, le jeune adulte peut être accompagné par un membre du Comité des usagers.

29. N. Lemieux et P. Lanctôt (1995). Commencer sa vie adulte à l'aide sociale. MSR, novembre.

30. C. Sylvestre et al. (1993). Expérimentation du programme d'encadrement personnalisé de la clientèle non participante. Évaluation d'impact. MMSRFP, février.

31. Depuis la publication du rapport, le MSSS a décidé de mettre en place un régime de co-assurance. Le montant additionné de la prime (non présente dans notre proposition), de la franchise et de la co-assurance dépasse largement le seuil recommandé dans notre rapport. Le régime s'applique aussi aux personnes présentant des contraintes sévères à l'emploi, contrairement à nos recommandations.

32. Les coupures dans le soutien au logement appliquées depuis la fin août 1996 ne faisaient évidemment pas partie de nos recommandations.

33. Certains commentateurs ont conclu faussement à une proposition de revenu minimum garanti de notre part. Le système PART ou APPORT repose sur une analyse individuelle des besoins et impose un test d'actif aux fins d'admission, ce qui exclut une large part de la population.

34. La proposition à l'effet d'étendre graduellement la PART à l'ensemble des personnes à faibles revenus a suscité des désaccords au sein du Comité externe de la réforme de la sécurité du revenu, ce qui explique en partie la publication de deux rapports distincts.

35. Cette question a aussi contribué aux désaccords entre les membres du Comité, les membres du rapport minoritaire exprimant, dans le cours des délibérations du Comité, un objectif de réforme à coûts nuls. Leur rapport propose de fait une réduction de l'enveloppe de la Sécurité du revenu. Au moment de l'écriture de ces lignes, le budget de l'aide sociale était fermé et rien ne laissait prévoir un changement d'orientation à cet égard même si l'on s'attend à ce que la récente réforme de l'assurance-chômage fasse encore une fois basculer des dizaines de milliers de personnes à l'aide sociale.

36. A. Noël (1996). "La droite et la gauche, le libéralisme et la social-démocratie». Conférence présentée lors du séminaire "L'État dans tous ses états», Centrale de l'enseignement du Québec, Magog. 